\title{
Postoperative Late-Onset Endophthalmitis Caused by Leishmania donovani: A Case Report
}

\author{
Bojan Kozomara $^{a} \quad$ Ernesta Potkonjak $^{a} \quad$ Ratimir Lazic $^{b} \quad$ Sanja Savicic \\ Nikica Gabric ${ }^{b}$ \\ aEye Clinic "Svjetlost," Banja Luka, Bosnia-Herzegovina; 'Special Eye Hospital "Svjetlost," \\ Zagreb, Croatia
}

\section{Keywords}

Cataract $\cdot$ Leishmania donovani $\cdot$ Pars plana vitrectomy

\section{Abstract}

Leishmania donovani is a human blood parasite that belongs to the genus Leishmania. We would like to present a case of late Leishmania donovani endophthalmitis in one eye of a patient that underwent simultaneous bilateral grade 3 cataract surgery.

(C) 2018 The Author(s)

Published by S. Karger AG, Basel

\section{Case Report}

A 77-year-old female patient was referred to our clinic for a routine, same-day, grade 3 cataract surgery on both eyes. Preoperatively and intraoperatively, the patient underwent standard preparation and surgical procedure. No visible endophthalmitis risk factors (blepharitis, conjunctivitis, keratitis) were seen on the preoperative exam. There were no intraoperative, nor early postoperative complications whatsoever. The follow-up ended 1 month after both surgeries without any evident signs of inflammation. At 3 months postoperatively, the patient was referred to us again complaining about rapid vision decrease in her right eye $(20 / 200)$, with redness and mild pain, while the left eye had no symptoms and retained 


\section{Case Reports in Ophthalmology}

excellent postoperative vision $(20 / 20)$. On examination, the presence of endothelial precipitates was noted, followed by a 2-mm hypopyon in the anterior chamber. Pupil was constricted and covered with a thick fibrotic membrane, which was also shown on the B-scan ultrasound exam in vitreous cavity. An urgent pars plana vitrectomy was performed. Vitreous tap was taken intraoperatively and sent for cytology and microbiology assessment. Cytology findings showed a large number of microorganisms in the cytoplasm of the phagocytes, which appeared to be Leishmania donovani parasites. The sample was taken according to the microbiology standards, in sterile conditions, and sealed in the specimen container. Therefore, the external contamination of the sample was ruled out. The patient was put on oral antiparasitic therapy (amphotericin B), as well as the local topical therapy (Tobradex ${ }^{\circledR}$ ) 5 times per day for 2 weeks. One month after pars plana vitrectomy, visual acuity improved to $20 / 60$, but the patient did not want to undergo a thorough examination at the clinic for infectious diseases.

\section{Discussion}

Endophthalmitis still represents a postoperative complication that can cause severe damage to the patient's visual performance. Leishmania is the obligate intracellular protozoan parasite which causes various forms of leishmaniasis. Their primary hosts are hyraxes, canids, rodents, and humans. It currently affects 6 million people in 98 countries. Even though a lot of preoperative, intraoperative, as well as postoperative measures are being taken every day in order to avoid it, endophthalmitis still occurs mostly in an acute form [1]. When it comes to late-onset endophthalmitis, it can be seen after gastrointestinal [2] and pacemaker surgeries [3]. Some authors even report an orbital involvement of post-cataract-glaucoma surgery endophthalmitis [4].

A variety of microorganisms can cause endophthalmitis, but their diversity is best described by Pluquet et al. [5], who report a novel species of microorganism that was isolated in a case of acute endophthalmitis. To our knowledge, this is the first case of late-onset endophthalmitis caused by Leishmania donovani. Currently, only Pradhan et al. [6] reported two cases of keratitis after post-kala-azar dermal leishmaniasis. Since the microorganism is being transferred by animals onto humans, we cannot know for certain how our patient got infected. Our theory is that the infestation might be systemic and that the eye was infected hematologically. Even still, the patient refused a thorough exam at the clinic for infectious diseases. Therefore, we cannot be certain about this fact.

Treatment options are the same in all endophthalmitis cases. An urgent pars plana vitrectomy should be performed as soon as possible. Postoperative visual acuity mostly depends on the timing of pars plana vitrectomy, while the causative agent also plays an important role. Bacterial agents usually have the best prognosis; on the other hand, parasitic and fungal microorganisms have the worst. Systemic therapy can contribute to a positive postoperative effect of the disease and should be included even before the pars plana vitrectomy, especially if there is proof of the causative agent.

In conclusion, we can say that endophthalmitis can still surprise us with the involved microorganisms even if we know more about the disease and the treatment options than ever before. Patient's cooperation is crucial for successful and on-time treatment, particularly in determining the causative agent. 


\section{Statement of Ethics}

Subjects have given their written informed consent to publish their case.

\section{Disclosure Statement}

The authors declare no conflicts of interest.

\section{References}

1 Shailaja S, Kamath Y, Hazarika M, Vishwanath S. Acute post-traumatic endophthalmitis secondary to Propionibacterium acnes. BMJ Case Rep. 2013 Oct;2013:bcr2013201452.

2 Wood M, Wright D, Witherspoon P. Fungal endophthalmitis: an unusual complication of GI surgery and endoluminal vacuum therapy. BMJ Case Rep. 2015 Jul;2015:bcr2015209902.

3 Pride P, Nutaitis M, L Charity P. Endophthalmitis: an unusual presentation of bacteremia. Am J Med Sci. 2013 Jan;345(1):70-1.

4 Cagini C, Iannone A, Fiore T, Lupidi M, Spadea L. Post-operative endophthalmitis caused by Acremonium falciforme with orbital and extra-orbital involvement following combined cataract and glaucoma surgery: a case report. J Med Case Reports. 2014 Nov;8(1):373.

5 Pluquet E, Brémond-Gignac D, Milazzo S, Mammeri H. Unusual acute endophthalmitis due to an as yet unclassified Acinetobacter gyllenbergii-like isolate. J Med Microbiol. 2011 Sep;60(Pt 9):1379-82.

6 Pradhan A, Basak S, Chowdhury T, Mohanta A, Chatterjee A. Keratitis after post-Kala-Azar dermal Leishmaniasis. Cornea. 2018 Jan;37(1):113-5. 